\title{
A new small nano-Kelvin resolution thermometer for low temperature experiments
}

\author{
Paul Welander, M. Barmatz, and Inseob Hahn \\ Jet Propulsion Laboratory \\ California Institute of Technology \\ 4800 Oak Grove Dr, Pasadena, CA 91109 , USA \\ hahn@squid.jpl.nasa.gov
}

\begin{abstract}
A small, high resolution paramagnetic susceptibility thermometer was developed using a $\mathrm{GdCl}_{3}$ paramagnetic salt. The device uses a SQUID (Superconducting Quantum Interference Device) magnetometer to determine the temperature dependent magnetization of the salt in a magnetic field. The magnetic field is provided by a pair of small samarium cobalt permanent magnet disks situated inside the thermometer housing. This eliminates the need for a heavy, charging solenoid used in a conventional SQUID based magnetic thermometer system. This thermometer can resolve approximately $10^{-9} \mathrm{~K}$ near the liquid-gas critical point of ${ }^{3} \mathrm{He}(\sim 3.31 \mathrm{~K})$. The drift rate of the thermometer was measured to be $<2 \times 10^{-13} \mathrm{~K} / \mathrm{s}$. This light weight thermometer $(\sim 7 \mathrm{~g})$ is a candidate for use in future low temperature Space Shuttle and International Space Station experiments. The present thermometer design easily permits a variety of magnetic materials for use in other applications.
\end{abstract}

\section{Introduction}

The high resolution thermometer (HRT) has been key to the success of low temperature experiments $[1,2]$. Its principle of operation is based on the strong temperature dependence of the magnetization of a paramagnetic salt placed in a magnetic field. The temperature resolution of this type of thermometer is typically better than $10^{-9} \mathrm{~K}$ and has a long term drift rate of $10^{-14} \mathrm{~K} / \mathrm{s}$ under optimized operating conditions. The conventional HRT system uses a long aspect ratio cylindrical superconducting tube to trap the required magnetic field. A superconducting solenoid is used to generate this field. This conventional HRT system is approximately $0.3 \mathrm{~m}$ long and weighs about $10 \mathrm{~kg}$. Although this technology is highly successful, the conventional design has several shortcomings that may preclude its future use in space.

Future low temperature experiments on the Low Temperature Microgravity Physics Facility (LTMPF) on the International Space Station (ISS) will have stringent experimental space and weight limitations. These low temperature experiments will typically last up to 6 months. The presence of a charging solenoid introduces a parasitic heat leak which will degrade the life time of the experiment. The long aspect ratio of the conventional HRT will cause excessive heating during a launch and require stronger supporting structures and thermal links.

A small HRT (sHRT) was developed to overcome these shortcomings. The sHRT weighs $\sim 7 \mathrm{~g}$ and is $\sim 3 \mathrm{~cm}$ long. It uses small permanent magnets to generate the required magnetic field. The permanent magnets eliminate the use of a heavy superconducting solenoid and allows the sHRT to fit easily into a limited experimental space. In this paper we present the thermometer design and its performance in the temperature range from 2 to $4 \mathrm{~K}$.

\section{Design}

The basic principle of a high resolution magnetic susceptibility thermometer is shown in Fig. 1. The current flowing through a superconducting loop will depend on the magnetization of the salt pill. This loop consists of a coil wrapped around the salt pill in series with the input coil of a SQUID. A temperature change will result in a change in the loop current, which in turn is measured by the SQUID magnetometer.

The conventional HRT design uses a long aspect ratio superconducting flux tube. The flux tube traps the required dc field and shields the superconducting sensing coil from external electromagnetic interference. In the new thermometer design the need for the flux tube and a separate charging solenoid is eliminated. Figure 2 shows a schematic drawing of the sHRT. The temperature sensitive element is a gadolinium trichloride salt $\left(\mathrm{GdCl}_{3}\right)$ situated in a beryllium 


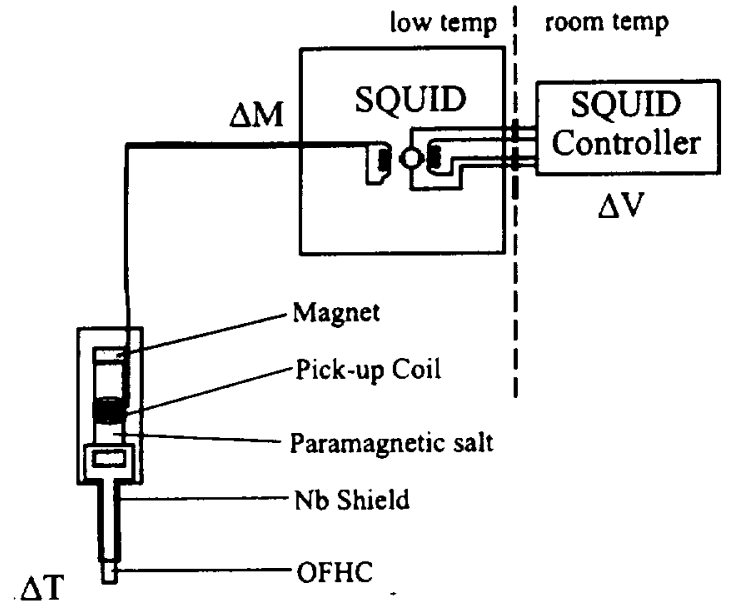

Figure 1. Schematic drawing of the high resolution thermometer system.

copper $(\mathrm{Be}-\mathrm{Cu})$ container. The thermal link to the salt was accomplished by machining numerous chimney-shaped fins from a high purity copper block. Relatively poor electrical conducting Be-Cu was chosen to minimize the Johnson noise $[3,4]$. The space between the fins was filled with $\mathrm{GdCl}_{3}$ by slowly crystallizing the molten salt in a quartz tube. Since the $\mathrm{GdCl}_{3}$ salt is very hygroscopic, the system had to be tightly sealed. The salt container was retrieved from the quartz tube, and quickly sealed using a copper cap containing a magnet. A low temperture epoxy $y^{1}$ was used to seal the container.

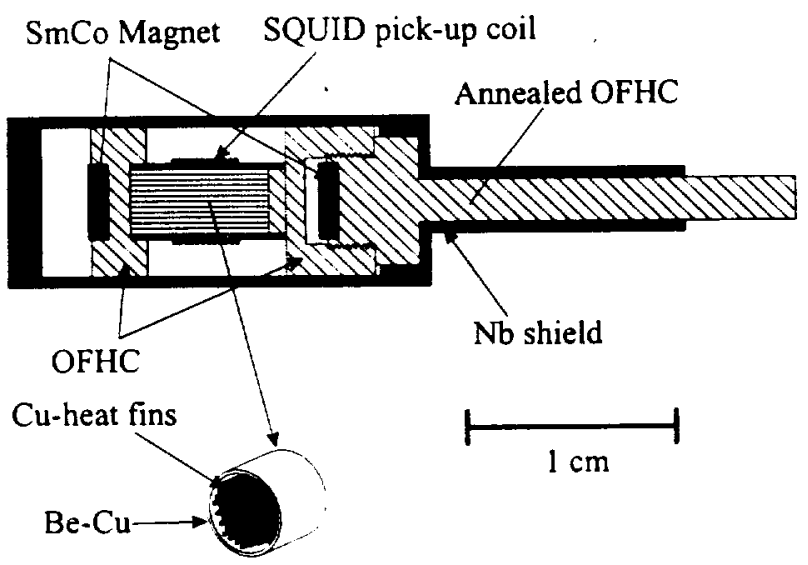

Figure 2. Schematic drawing of the new sHRT.

The magnets were located such that the strength of the magnetic field near the salt was roughly $200 \mathrm{Oe}$. The su-

\footnotetext{
'Stycast 1266 from Emerson and Cuming Inc., Canton. Massachusetts, USA.
}

perconducting $\mathrm{Nb}$ housing shielded the pick-up coil from the ambient field. A SQUID pick-up coil ( $\mathrm{Nb}-\mathrm{Ti}$ wire) was wound on $\mathrm{Be}-\mathrm{Cu}$ container. The value of the inductance of the coil was determined to match a dc-SQUID sensor ( $1.2 \mu \mathrm{H})$ to maximize sensitivity. The coil leads were twisted and fed into the superconducting $\mathrm{Nb}$ capillary. Epoxy ${ }^{1}$ was injected into the capillary to minimize microphonic pick-up. The OFHC (Oxygen Free High conductivity Copper) was used to thermally link the salt to the outside. To increase the thermal conductivity, the copper rod was oxygen annealed. The measured RRR (residual resistance ratio) was roughly 2000 . The rod was cooled using liquid nitrogen and press fit into the lower body of the $\mathrm{Nb}$ shield. The magnet was then glued to the rod and screwed into the main salt container. Total mass of the thermometer is about $7 \mathrm{~g}$.

\section{Test and Results}

The sensitivity and noise performance of the new thermometer was tested in a low temperature cryostat. The thermometer was mounted on a helium filled container. The large heat capacity and fast thermal response of superfluid helium made the container an ideal thermal reservoir for the test. The container was surrounded by a thermal radiation shield stage that was connected to secondary thermal isolation stages. A helium evaporation cooler was thermally linked to the stages. Each stage was carefully temperature controlled to achieve a stable thermal environment for the test. The SQUID sensor (Quantum Design Inc.) was mounted inside the vacuum can and temperature controlled near $4.2 \mathrm{~K}$. A high permeability metal shield surrounded the dewar to minimize any ambient trapped field in the thermometer system. A calibrated germanium resistance thermometer was used to measure the temperature of the container. The system was cooled slowly below $2 \mathrm{~K}$ while monitoring the thermometer magnetization $(\mathrm{M})$ and the temperature (T) of the helium filled container. Figure 3 shows the sensitivity of the thermometer $(\Delta \mathrm{M} / \Delta \mathrm{T})$ in units of flux quanta per $\mu \mathrm{K}$. The sharp singularity in the curve near 2.2 $\mathrm{K}$ indicates the ferromagnetic transition of the $\mathrm{GdCl}_{3}$ salt.

The white noise level of the dc-SQUID sensor and its electronics is typically $\mu \Phi_{0} / \sqrt{H z}$. This implies an ideal temperature resolution of $\sim 0.5 \mathrm{pK} / \sqrt{\mathrm{Hz}}$ at $3.3 \mathrm{~K}$. However. the actual thermometer resolution is usually limited by thermodynamic fluctuations in the temperature sensor, not by read-out electronics. The measured temperature resolution of sHRT was $\sim \ln K$ in a $1 \mathrm{~Hz}$ bandwidth near $3.3 \mathrm{~K}$. The drift rate of the thermometer was also measured relative to an almost identical second HRT by monitoring the output of one thermometer while controlling the temperature of the reservoir with the second thermometer. Although the measurement apparatus was not ideal for ultimate performance testing, the measured relative drift rate between thermome- 


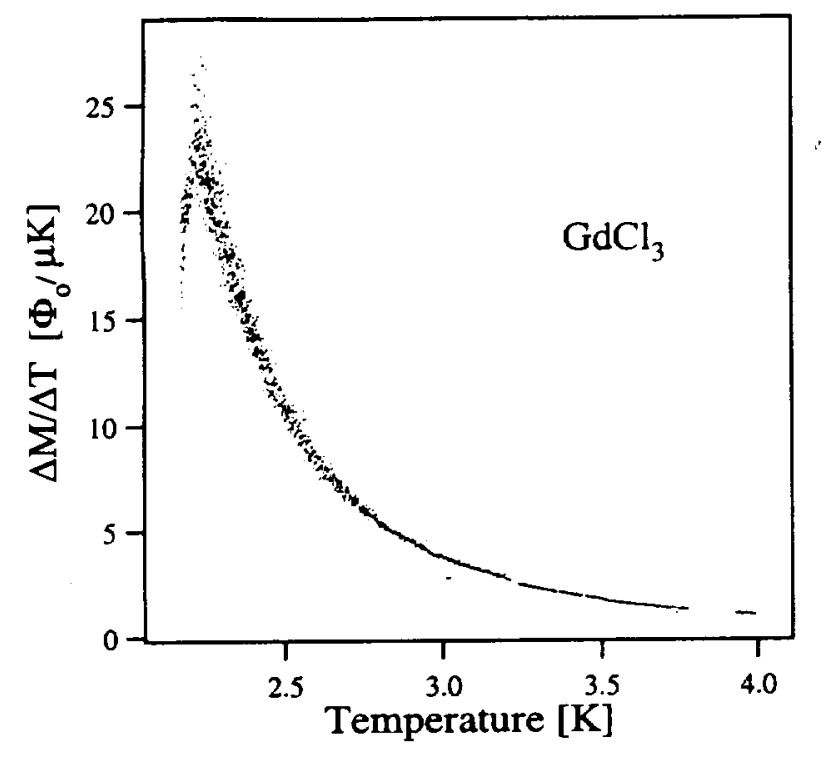

Figure 3. Sensitivity of the sHRT.

ters was $<2 \times 10^{-13} \mathrm{~K} / \mathrm{s}$.

The noise power spectral density of the SHRT was also measured at a temperature very close to the ${ }^{4} \mathrm{He}$ superfluid transition temperature $(T=2.17 K)$. To measure the noise power spectral density, the reservoir temperature was slowly drifted toward a constant shield stage temperature. The output of the thermometer was then recorded and analyzed using a Fast Fourier Transform (FFT). The linear drift was subtracted before the FFT analysis. The result of the analysis is shown by the solid line in Fig. 4. Total root-mean-square (rms) temperature noise between $\mathrm{dc}$ to $10 \mathrm{~Hz}$ was $\sim 0.14 \mathrm{nK}$ while the rms noise between dc to 1 $\mathrm{Hz}$ was $\sim 0.11 \mathrm{nK}$. The noise spectral density of a HRT can be described by an equation derived from the fluctuationdissipation thereom [5]. A simple thermal model of the HRT corresponds to a salt with thermal mass (heat capacity, C) connected to a reservoir through a thermal link (thermal resistance, $\mathrm{R}$ ). The time constant for this model is $\tau=R C$. The current sHRT noise spectrum can not be well understood by a single time constant. However, the general feature of the noise spectral density in Fig. 4 (solid line) is comparable to that obtained by Qin et.al. [6] for conventional HRTs (dashed lines). One conventional thermometer was used in Lambda Point Experiment (LPE) and a further improved HRT was used in the Confined Helium Experiment $(\mathrm{CHeX})$, both of which were flown on the Shuttle. ${ }^{2}$

\footnotetext{
2Both thermometers used Copper Amonium Bromide (CAB) as a paramagnetic salt
}

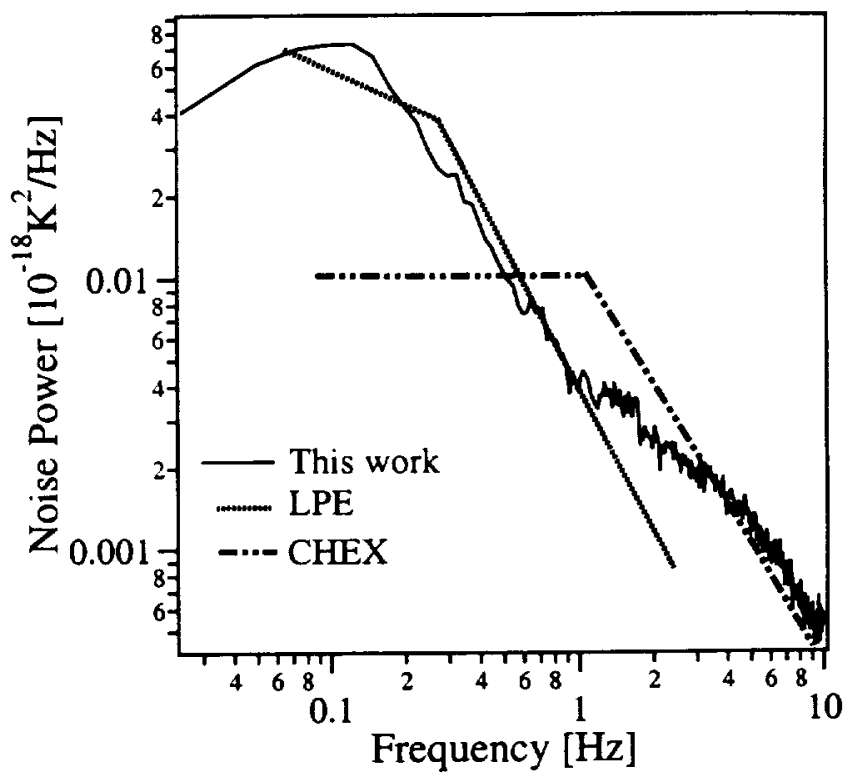

Figure 4. Noise power spectral density of the thermometer at $\mathrm{T}=2.17 \mathrm{~K}$.

\section{Summary}

A small high resolution thermometer (sHRT) was fabricated and tested. The device showed better than $1 \mathrm{nK}$ temperature resolution in a $1 \mathrm{~Hz}$ bandwidth below $3.3 \mathrm{~K}$. The relative drift rate of the thermometer was $<2 \times 10^{-13} \mathrm{~K} / \mathrm{s}$. In the future, the absolute drift rate will be measured using the superfulid helium transition as a fixed temperature point indicator. Noise spectral density was also measured at $2.17 \mathrm{~K}$. The result shows that the performance of the sHRT is comparable to the conventional HRT. This sHRT is designed for easy replacement of the paramagnetic salt. This permits the present design to be used in other temperature ranges and applications. Further miniaturization of this sHRT using a different salt $\left(\mathrm{La}_{x} \mathrm{Gd}_{1-x} \mathrm{Cl}_{3}\right)$ for lower temperature application is currently under development. Due to its compact size and reliable performance, this thermometer is being considered for use in a future microgravity low temperature experiment in Earth orbit [7].

We thank P. Day for useful discussion. This work is supported by the NASA Advanced Technology Development program.

\section{References}

[1] J. Lipa, D. Swanson, J. Nissen. T. Chui. and U. Israelsson. Phys. Rev. Lett. 76:944, 1996. 
[2] J. Lipa, D. Swanson, J. Nissen, P. Williamson, Z. Geng, D. Stricker, T. Chui, U. Israelsson, M. Larson Joumal of Low Temperature Physics, 113:849, 1998.

[3] H. Fu, H. Baddar, K. Kuehn, M. Larson, N. Mulders, A. Schegolev, and G. Ahlers Journal of Low Temperature Physics, 111:49, 1998.

[4] P. Day, I. Hahn, T. Chui, A. W. Harter, D. Rowe, and J. Lipa. Joumal of Low Temperature Physics, 107:309, 1997.

[5] T.C.P. Chui, D. Swanson, M.J. Adriaans, J.A. Nissen, and J.A. Lipa Phys. Rev. Lett, 69:3005, 1992.

[6] X. Qin, J.A. Nissen, D. Swanson, P.R. Williamson, D. Stricker, J. Lipa, T. Chui, and U. Israelsson. Czech. J. Phys. Suppl, 46:2857, 1997.

[7] M. Barmatz, I. Hahn, and F. Zhong. Journal of Low Temperature Physics, 113:891, 1998. 